association between antibiotic use and current chlamydia infection through logistic regression.

Results Among 391 recruited women with a mean age of $31.51 \pm 6.20$ years, $41.2 \%(161 / 391)$ of participants selfreported that they used antibiotics in recent 12 months, and the prevalence of chlamydia incidence was $8.2 \%$ (32/ 391). The $\beta$-lactam antibiotics (Penicillin, Amoxicillin, ceftriaxone) were the most popular $(89.4 \%, 144 / 161)$ among female patients, while the rate of using other antibiotics (Clindamycin, Metronidazole) was the lowest (31.7\%,51/ 391). Additionally, we found that age, histories of pelvic inflammatory disease and reproductive infections, and had clinical symptoms in the recent 12 months were significantly associated with antibiotic usage among RAW. Overall antibiotic use (aOR: 0.32; 95\%CI: 0.15-0.94) and using antibiotics under the Access classification (aOR:0.30; 95\% CI: $0.07-0.92$ ) were negatively associated with current chlamydia infection.

Conclusion This study suggests that a high rate of recent antibiotic use and an inverse association between the antibiotic use and current chlamydia infection among RAW in Guangdong, China. Findings will provide an insight to evaluate the impact of antibiotic use on chlamydia screening.

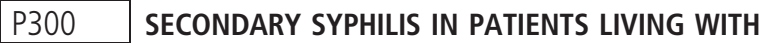 AND WITHOUT HIV IN CALI, COLOMBIA: AN IMMUNOHISTOCHEMISTRY STUDY}

${ }^{1} \mathrm{M}$ Amórtegui ${ }^{*}$, 1,2J García Luna, ${ }^{3} \mathrm{M}$ Fiel-Gan, ${ }^{1,2} \mathrm{~L}$ Ramírez, ${ }^{4,5} \mathrm{~K}$ Hawley, ${ }^{3} \mathrm{P}$ Newcom, 1,4,5,6 J Salazar. 'Centro Internacional de Entrenamiento e Investigaciones Médicas (CIDEIM), Cali, Colombia 2 Universidad ICESI, Cali, Colombia. 3 Department of Pathology, Hartford Hospital, Hartford, CT, USA. 4 Department of Pediatrics, University of Connecticut School of Medicine, Farmington CT, USA. 5 Division of Infectious Diseases, Connecticut Children's, Hartford CT, USA. 6 Department of Immunology, University of Connecticut School of Medicine, Farmington CT, USA., Cali, Colombia; '2Universidad ICESI, Cali, Colombia; ${ }^{3}$ Department of Pathology, Hartford Hospital, Hartford, USA; ${ }^{4}$ Department of Pediatrics, University of Connecticut School of Medicine, Farmington, USA; ${ }^{5}$ Division of Infectious Diseases, Connecticut Children's, Hartford, USA; ${ }^{6}$ Department of Immunology, University of Connecticut School of Medicine, Hartford, USA

\subsection{6/sextrans-2021-sti.358}

Background The upsurge of syphilis cases is a public health threat, particularly in people living with HIV (PLWH). The aim of this case series was to describe the histological and immunohistochemical changes in skin lesions of patients with secondary syphilis (SS) by HIV status.

Methods Clinical data and skin biopsies from SS patients who were consented and enrolled in Cali, Colombia between 2003 and 2018 were included in the analysis. Clinical data was extracted using a standardized form. Four-micrometer sections of paraffin embedded skin biopsies were stained with haematoxylin and eosin (H\&E) or labelled with antibodies to detect CD4, CD8, CD56, CD68, CD138 or Treponema pallidum (Tp). Data from PLWH and people without HIV (PWH) were compared using the Fisher's exact test for qualitative variables, the Student's t test or the Wilcoxon rank-sum test for quantitative variables according to their distribution. The KruskalWallis test was used to compare cellular populations according to categories of $\mathrm{Tp}$ burden (absent, sparse or moderate to severe).

Results Of 114 subjects with SS, 28 PWH and 10 PLWH subjects had available skin biopsies. Most participants were male
(65.8\%), the mean age was 27 years (range 18-60). PLWH were more likely to identify themselves as mestizo $(100 \%$ vs $52 \%, \mathrm{p}=0.01)$, there were no differences in age, sex, symptoms or signs of syphilis. PLWH were more likely to have acanthosis $(70 \%$ vs $32 \%, \mathrm{p}=0.06)$ and epidermal hyperplasia $(70 \%$ vs $18 \%, \mathrm{p}=0.005)$. There were no differences in other H\&E findings. PLWH had lower CD4 cells in the skin $(p=0.0001)$, without differences in other cellular populations or $\mathrm{Tp}$ positivity. There were no differences in the cell counts by categories of $\mathrm{Tp}$ burden.

Conclusions HIV infection was associated with non-specific epidermal hyperplasia and lower CD4 cell counts in the skin, but not with $\mathrm{Tp}$ positivity.

\section{P301 ELIMINATION OF MOTHER-TO-CHILD TRANSMISSION OF HIV AND SYPHILIS IN THE MERCOSUL COUNTRIES: A GENERAL OVERVIEW}

M Aragon*, C Alves, P Gaspar, G Pereira, A Miranda. Ministry of Health of Brazil, Srtvn Quadra 701, Lote D, Edifício Po700 - 5ªndar, Cep70719-040, Brasilia, Brazil

\subsection{6/sextrans-2021-sti.359}

Background Mercosul (Southern Common Market) consists of four countries - Argentina, Brazil, Paraguay and Uruguay with close economic relations in Latin America. The displacement of people within countries and across borders is frequent observed and needs surveillance and health care implementation for infections that do not recognize borders. The present study aims to presenting the HIV and syphilis overview in Mercosul countries, highlighting the vertical transmission of these diseases.

Methods We systematically collected data from Mercosul countries in the past two years through Ministry of Health websites, countries national official data and Pan-American Health Organization (PAHO) reports. We compared country data with each other and with the targets set by PAHO elimination of mother-to-child transmission (MTCT) of HIV and syphilis.

Results The HIV detection in Uruguay and Paraguay had the highest rates, with 29/100,000 inhabitants and 22.8/100,000 inhabitants, when compared to Brazil (17.8/100,000 inhabitants) and Argentina (10.3/100,000 inhabitants). Regarding the MTCT of HIV, Brazil and Uruguay are within the PAHO elimination target $(\leq 2 \%)$, showing rates of $1.6 \%$ and $1.5 \%$, respectively. The syphilis rates have increased significantly in recent years, reaching 75.8/100,000 inhabitants in Brazil, 51.1/100,000 inhabitants in Argentina and 46/ 100,000 inhabitants in Paraguay. In Brazil, the pregnant rates were 20.8 pregnant women per 1,000 live births (LB) in 2019. The goal of eliminating congenital syphilis $(\leq 0.5 /$ 1,000 LB) was not achieved by any country, with Paraguay having the highest rate $(17.9 / 1,000 \mathrm{LB})$, followed by Brazil $(8.2 / 1,000$ LB), Argentina $(1.55 / 1,000$ LB) and Uruguay (1.4/1,000 LB).

Conclusions Official national standardization data is an important tool for planning joint actions. These data indicate the need to maximize strategies, especially related to congenital syphilis prevention. Also they reinforce the importance of political commitment from the Ministries of Health and international cooperation for the elimination of MTCT in Mercosul. 